\title{
Gravitational spin-orbit coupling in binary systems, post-Minkowskian approximation and effective one-body theory
}

\author{
Donato Bini ${ }^{1}$ and Thibault Damour ${ }^{2}$ \\ ${ }^{1}$ Istituto per le Applicazioni del Calcolo "M. Picone", CNR, I-00185 Rome, Italy \\ ${ }^{2}$ Institut des Hautes Etudes Scientifiques, 91440 Bures-sur-Yvette, France
}

(Dated: October 3, 2018)

\begin{abstract}
A novel approach for extracting gauge-invariant information about spin-orbit coupling in gravitationally interacting binary systems is introduced. This approach is based on the "scattering holonomy", i.e. the integration (from the infinite past to the infinite future) of the differential spin evolution along the two worldlines of a binary system in hyperboliclike motion. We apply this approach to the computation, at the first post-Minkowskian approximation (i.e. first order in $G$ and all orders in $v / c$ ), of the values of the two gyrogravitomagnetic ratios describing spin-orbit coupling in the Effective One-Body formalism. These gyrogravitomagnetic ratios are found to tend to zero in the ultrarelativistic limit.
\end{abstract}

\section{INTRODUCTION}

The Effective One-Body (EOB) formalism was conceived [1-4] with the aim of analytically describing both the last few orbits of, and the complete gravitationalwave signal emitted by, coalescing binary black holes. The EOB formalism played a key role in allowing one to compute, in a semi-analytic way, hundreds of thousands of templates which have been used to search for, and analyze, the recently detected gravitational wave signals from coalescing binary black holes [5-7]. Any theoretical advance in EOB theory might benefit to the burgeoning field of gravitational wave astronomy. The present work will introduce a new approach to the theoretical description of spin-orbit couplings within the EOB formalism, and apply it to the computation, to the first post-Minkowskian (1PM) approximation (i.e. first order in $G$ but all orders in $v / c$ ), of the values of the two gyrogravitomagnetic ratios, $g_{S}$ and $g_{S_{*}}$, which describe spin-orbit coupling in EOB theory.

The EOB formalism was originally developed within the post-Newtonian $(\mathrm{PN})$ approximation method [1-4] . Recently, a novel approach to EOB theory, based on the post-Minkowskian (PM) approximation method, has been introduced [8]. The PM approximation scheme (expansion in powers of $G$, for fixed velocities) physically correspond to small-angle scattering in hyperboliclike encounters (with arbitrary incoming velocities) 9 16]. Ref. [8] has applied this approach to the orbital dynamics of a system of two nonspinning compact bodies. The aim of the present paper is to generalize this approach to the case of gravitationally interacting spinning bodies, considered in the pole-dipole approximation, i.e. described by two masses $\left(m_{1}, m_{2}\right)$ and two spin 4 -vectors $\left(s_{1}, s_{2}\right)$. We will work linearly in the spins, and consider (as in [8]) hyperboliclike motions.

When considering nonspinning binary systems, the prime observable consequence of the orbital dynamics is the scattering angle $\chi$, measured in the center-of-mass (c.m.). More precisely, as emphasized in Refs. [17, 18], the orbital dynamics is encoded, in a gauge-invariant manner, in the functional link between $\chi$ and the total (c.m.) energy, $\mathcal{E}_{\text {real }}$, and (c.m.) orbital angular momentum, $L$, of the system. When considering spinning systems, with parallel spins, one has to deal with a more general (gauge-invariant) functional link, namely $\chi=\chi\left(L, S_{1}, S_{2}\right)$, where $S_{1}, S_{2}$ denote the algebraic magnitudes of the parallel spins [19]. [The orbital angular momentum, and the spin magnitudes are defined so that $J=L+S_{1}+S_{2}$ is equal to the (well-defined) total angular momentum of the system in the c.m. frame.] See Ref. 19] for recent high $\mathrm{PN}$-order results on the function $\chi\left(L, S_{1}, S_{2}\right)$.

Here we introduce an alternative approach to a gaugeinvariant characterization of the dynamics of spinning systems. Instead of being based on a scalar function $\left[\chi\left(L, S_{1}, S_{2}\right)\right]$ that is only defined for parallel spins, we will instead consider matrix-valued (gauge-invariant) observables: the "scattering holonomy," and the related "spin holonomy" (both being defined along each of the two infinite worldlines representing the spacetime history of the two bodies). These quantities will be defined in the following Sections. Before entering any technical detail, let us emphasize that a significant advantage (over the computation of $\left.\chi\left(L, S_{1}, S_{2}\right)\right)$ of our new method, is that we will get information about the linear-in-spin couplings from a calculation where we will be able to actually neglect spin-effects in the dynamics! This useful feature of our new approach is akin to the method used in Refs. [20, 21] to derive (within a PN framework) the spin-orbit terms in the two-body Hamiltonian from the metric generated by two nonspinning bodies.

In the present paper, we apply our approach to the computation of the spin-orbit couplings at the linear order in $G$ (1PM approximation). In addition, we show how to extend the dictionary between the real two-body dynamics and its EOB image so as to allow us to transcribe our explicit 1PM spin-scattering computation, into a corresponding 1PM-accurate knowledge of the two gyrogravitomagnetic ratios, $g_{S}$ and $g_{S_{*}}$ that describe spinorbit coupling in EOB theory.

We (generally) employ units where $c=1$; use the mostly plus signature for the spacetime metric; and use 
standard EOB notations, notably

$$
M \equiv m_{1}+m_{2} ; \mu \equiv \frac{m_{1} m_{2}}{m_{1}+m_{2}},
$$

with the symmetric mass ratio of the binary system being denoted as

$$
\nu \equiv \frac{\mu}{M}=\frac{m_{1} m_{2}}{\left(m_{1}+m_{2}\right)^{2}} .
$$

\section{NEW CONCEPT: SCATTERING HOLONOMY}

We consider the scattering of two, gravitationally interacting, spinning bodies. Geometrically, we deal with two worldlines, $\mathcal{L}_{1}$ and $\mathcal{L}_{2}$, in an asymptotically flat curved spacetime endowed with a metric $g=g_{\mu \nu} d x^{\mu} d x^{\nu}$ (generated by the energy tensor supported on the worldlines). $\mathcal{L}_{1}$ and $\mathcal{L}_{2}$ asymptote to straight lines in the infinite past and the infinite future. In our pole-dipole approximation, each worldline (say, $\mathcal{L}_{1}$ ) is a priori endowed with three 4 -vectors, namely : the 4 -velocity, $u_{1}=u_{1}^{\mu} \partial_{\mu}$, (with the usual unit normalization $u_{1} \cdot u_{1}=-1$ ), the 4-momentum $p_{1}=p_{1}^{\mu} \partial_{\mu}$ (with the normalization $p_{1} \cdot p_{1}=-m_{1}^{2}$ ), and a spin 4 -vector, $s_{1}=s_{1}^{\mu} \partial_{\mu}$ (constrained to satisfy $p_{1} \cdot s_{1}=0$, and $s_{1} \cdot s_{1}=s_{1}^{2}=$ cst.). [Such a 4 -vector $s_{1}$ is equivalent to an antisymmetric spin tensor $S_{1}^{\mu \nu}$ satisfying $S_{1}^{\mu \nu} p_{\nu}=0$. In a local Lorentz frame ${ }^{1}$ whose unit time vector is $p_{1} / m_{1}$, the (only nonvanishing) spatial components of $S_{1}$ are defined to be dual to the (only nonvanishing) spatial components of $s_{1}$ : $S_{1}^{\hat{\hat{i}} \hat{j}}=\epsilon^{\hat{i} \hat{j} \hat{k}} s_{1}^{\hat{k}}$.] The classic works (among others) of Mathisson, Papapetrou, Tulczyjew and Dixon (see [22] and references therein) have shown that the data $u_{1}, p_{1}, s_{1}$ (or $u_{1}, p_{1}, S_{1}$ ) satisfy a set of universal evolution equations. The latter equations imply, in particular, a link between $u_{1}$ and $p_{1}$ of the form

$$
p_{1}=m_{1} u_{1}+O\left(s_{1}^{2}\right) \text {. }
$$

Using this link, the differential evolution system for $p_{1}$ and $s_{1}$ has the form

$$
\frac{D_{g} p_{1 \mu}}{d \tau_{1}}=-\frac{1}{2} R_{\mu \nu \kappa \lambda} u_{1}^{\nu} S_{1}^{\kappa \lambda}
$$

and

$$
\frac{D_{g} s_{1}^{\mu}}{d \tau_{1}}=O\left(s_{1}^{2}\right)
$$

Here, $D_{g}$ denotes the covariant derivative (along the worldline) associated with $g=g_{\mu \nu} d x^{\mu} d x^{\nu}$, and $R_{\mu \nu \kappa \lambda}$

\footnotetext{
${ }^{1}$ In a general frame $S_{1}^{\mu \nu}=m_{1}^{-1} \eta^{\mu \nu \kappa \lambda} p_{1 \kappa} s_{1 \lambda}$, with the appropri-
} ate definition of the Levi-Civita tensor $\eta$. the corresponding curvature tensor (with the usual sign convention that $R_{1212}>0$ on a 2 -sphere).

Because we work linearly in spins, and because the method we shall introduce extracts the spin-orbit coupling only from the evolution of $s_{1}$ along $\mathcal{L}_{1}$, we are allowed (as will be clear from our formulas below) to neglect the $O\left(s_{1}\right)$ spin-curvature coupling in the evolution of $p_{1}=m_{1} u_{1}+O\left(s_{1}^{2}\right)$, and use a simplified evolution system amounting to stating that $p_{1}=m_{1} u_{1}$, and that $u_{1}$ and $s_{1}$ are parallely propagated (in $g_{\mu \nu}$ ) along $\mathcal{L}_{1}$ (with a corresponding statement for $u_{2}$ and $s_{2}$ along $\mathcal{L}_{2}$ ). Using an index-free notation, and working with differential forms, we will write

$$
D_{g} u_{1}=0=D_{g} s_{1},
$$

where

$$
D_{g}=d+\omega_{1} .
$$

Here, the differential $d$ is taken along $\mathcal{L}_{1}$, and $\omega_{1}$ denotes the evaluation along $\mathcal{L}_{1}$ of the Levi-Civita connection one-form $\omega$ acting on contravariant four-vectors. Henceforth, we generally think of $u_{a}, s_{a}(a=1,2), g, \omega$ as abstract geometric objects. These objects can then be expressed in terms of various types of components: e.g. coordinate-frame components when using a generic coordinate system, or moving-frame components when using such frames. In a coordinate frame the connection, acting on a contravariant four-vector (e.g. $u_{1}=u_{1}^{\mu} \partial_{\mu}$, or $\left.s_{1}=s_{1}^{\mu} \partial_{\mu}\right)$ becomes

$$
\omega_{\nu}^{\mu}=\Gamma_{\nu \lambda}^{\mu} d x^{\lambda}
$$

where

$$
\Gamma_{\nu \lambda}^{\mu}=\frac{1}{2} g^{\mu \sigma}\left(\partial_{\nu} g_{\lambda \sigma}+\partial_{\lambda} g_{\nu \sigma}-\partial_{\sigma} g_{\nu \lambda}\right)
$$

To close the evolution system (6), we need to replace $g_{\mu \nu}$ by the solution of Einstein's equations, with the corresponding pole-dipole source terms supported by the two worldlines. We also need, as is standard in perturbative approaches to the two-body problem, to regularize the formally infinite evaluations of $g$ and $\omega$ along the worldlines. [See Ref. 12] for a detailed discussion of these regularizations within the PM context.] Let us note in advance another simplifying feature of our approach. As we can consistently neglect the Mathisson-Papapetrou, $O\left(s_{1}\right)$, non-geodesic correction to $D_{g} u_{1} / d \tau_{1}$, we can also (when considering the generation of $g_{\mu \nu}$ by our spinning binary system) neglect the spin contributions to $g_{\mu \nu}$, i.e. consider that $g_{\mu \nu}$ is generated by two nonspinning point masses.

We now define the scattering holonomy, $\Lambda_{1}$, along $\mathcal{L}_{1}$ as the parallel-transport linear operator (acting on contravariant four-vectors) integrated along $\mathcal{L}_{1}$ from the infinite past to the infinite future. As a parallely transported vector $v$ satisfies

$$
d v=-\omega_{1} v
$$


(where $\omega_{1}$ is evaluated on $\mathcal{L}_{1}$ ) the $\mathcal{L}_{1}$ scattering holonomy reads ( $T$ denoting Dyson's time-ordered product [23])

$$
\begin{aligned}
\Lambda_{1} & =T_{\mathcal{L}_{1}}\left[e^{-\int \omega_{1}}\right] \\
& =1-\int_{-\infty}^{+\infty} \omega_{1}+\frac{1}{2} \int_{-\infty}^{+\infty} \int_{-\infty}^{+\infty} T\left[\omega_{1} \omega_{1}^{\prime}\right]+
\end{aligned}
$$

with a similar result along $\mathcal{L}_{2}$ given by exchanging $1 \leftrightarrow 2$. The time-ordered product in Eq. (11) refers to an integration performed along $\mathcal{L}_{1}$, from the infinite past to the infinite future. In Eq. (11), $\omega_{1}=\omega_{1}(t)=\sigma_{1}(t) d t, \omega_{1}^{\prime}=$ $\omega_{1}\left(t^{\prime}\right)=\sigma_{1}\left(t^{\prime}\right) d t^{\prime}$, and $T\left[\omega_{1} \omega_{1}^{\prime}\right]=\sigma_{1}\left(t_{>}\right) \sigma_{1}\left(t_{<}\right) d t d t^{\prime}$, with $t_{<}=\min \left(t, t^{\prime}\right)$ and $t_{>}=\max \left(t, t^{\prime}\right)$ denotes the timeordered product of two integrands coming from the formal expansion of the exponential in the first line. Here, $t$ denotes any time-related parameter along $\mathcal{L}_{1}$. [Note that our differential-form formulation does not depend on the choice of any specific parametrization along the worldline.]

The scattering holonomy, $\Lambda_{1}$, is a linear operator mapping the (abstract) vector space of contravariant fourvectors at $-\infty$ (along $\left.\mathcal{L}_{1}\right)$ onto its analog vector space at $+\infty$. As we describe here the scattering motions of an isolated system, the two latter asymptotic spaces of fourvectors can be naturally identified with the vector space of Minkowski four-vectors. [We use here asymptotic flatness.] In other words, if we use a coordinate system that respects manifest asymptotically flatness (as will be the case in our PM computation), the scattering holonomy computed as in Eq. (11) will concretely be a $4 \times 4$ matrix $\Lambda_{1}{ }^{\mu}{ }_{\nu}$ acting on Minkowski vectors.

It will be henceforth convenient to denote by a subscript - (respectively + ) asymptotic quantities at $-\infty$ (resp. $+\infty)$. The scattering holonomy is a linear map between $u_{1}^{-}, s_{1}^{-}$and $u_{1}^{+}, s_{1}^{+}$:

$$
u_{1}^{+}=\Lambda_{1} u_{1}^{-} ; s_{1}^{+}=\Lambda_{1} s_{1}^{-} .
$$

This linear operator is geometrically defined and therefore, because of asymptotic flatness, gauge-invariantly defined. [Concretely the matrix $\Lambda_{1}{ }_{\nu}{ }_{\nu}$ is invariant under coordinate diffeomorphisms that decay sufficiently fast at infinity so as to respect manifest asymptotic flatness.] $\Lambda_{1}$ is a classical scattering operator which describes the mapping between the incoming momentum ${ }^{2}$, $p_{1}^{-}=m_{1} u_{1}^{-}$and spin $s_{1}^{-}$, and the corresponding outgoing ones, $p_{1}^{+}=m_{1} u_{1}^{+}$and $s_{1}^{+}$. Note that it contains in particular the information about the usual scattering angle $\chi$. As we have neglected the spin corrections to the evolution of $u_{1}$, our present estimate of $\Lambda_{1}$ only describes (when acting on $u_{1}^{-}$) the orbital part, $\chi_{\text {orb }}(L)$, of the scattering angle. However, we shall see how to extract spin-orbit information from the action of $\Lambda_{1}$ on spacelike vectors.

2 The curvature-related $O\left(s_{1}^{2}\right)$ general difference between $p_{1}$ and
As we said, all the asymptotic four-vectors $u_{a}^{ \pm}, s_{a}^{ \pm}$ $(a=1,2)$ live in an asymptotic Minkowski space. Moreover, as parallel transport preserves the length, the two $\Lambda_{a}$ 's preserve the asymptotic flat metric. In other words, the two matrices $\Lambda_{a}^{\mu}{ }_{\nu}$ are usual Lorentz transformations belonging to $S O(3,1)$, and preserving $\eta_{\mu \nu}=$ $\operatorname{diag}(-1,+1,+1,+1)$.

\section{III. "SPATIAL" SPIN VECTORS}

The covariant spin four-vectors $s_{1}, s_{2}$ are not the usual, canonical, spatial spin three-vectors $\mathbf{S}_{1}, \mathbf{S}_{2}$ (with constant Euclidean lengths) that enter the Hamiltonian description of binary dynamics (such as the EOB description). A simple way of constructing spatial spin threevectors associated with the four-vectors $s_{a}$ (which are orthogonal to the tangent $u_{a}$ to $\left.\mathcal{L}_{a}\right)^{3}$ has been given in Ref. 20]. It can be described geometrically in the following way. Given two (future-directed) unit timelike vectors $u$ and $v$ in the tangent space of some point in a Riemannian manifold, there is a unique local Lorentz transformation, say $B_{g}(u \rightarrow v)$ (where the letter $B$ stands for "boost"), which acts in the 2-plane spanned by $u$ and $v$ so as to rotate $u$ into $v$, while leaving invariant the complementary 2-plane orthogonal to $u$ and $v$. [We give below the explicit expression of the $4 \times 4$ boost matrix $\left[B_{g}(u \rightarrow v)\right]_{\nu}^{\mu}$ in the case where the metric $g$ is flat.] With this notation in hand, and given a global field of (future-directed) unit time-vectors $U=U^{\mu} \partial_{\mu}$ orthogonal to the time-slicing that we use to describe our spacetime ${ }^{4}$, the spatial spin vector associated with the four-vector $s_{1}$ is defined, at each point $x_{1} \in \mathcal{L}_{1}$, by (actively) applying the boost operator $B_{g}\left(u_{1} \rightarrow U\right)$, acting in the tangent vector space at the point $x_{1}$. Note that $B_{g}\left(u_{1} \rightarrow U\right)$ is defined so that it rotates $u_{1}$ into $U$, i.e. it is the inverse of the boost that would map the lab frame associated with $U$ into the local rest-frame of $\mathcal{L}_{1}$. We have indicated in subscript that this linear map is locally defined in a curved spacetime with metric $g$ (evaluated at point $x_{1}$ ).

We then define ${ }^{5}$

$$
S_{1}=B_{g}\left(u_{1} \rightarrow U\right) s_{1} ; S_{2}=B_{g}\left(u_{2} \rightarrow U\right) s_{2},
$$

where the abstract four-vectors $S_{1}, S_{2}$ now live in the corresponding local three-planes orthogonal to $U\left(x_{1}\right)$, $U\left(x_{2}\right)$. See Fig. 1, which assumes that the vector field $U$ is globally orthogonal to a spacelike hypersurface, as is the case in the Hamiltonian formalism where spacetime is foliated (in the c.m. frame) by $t=$ cst. hypersurfaces. As

\footnotetext{
3 At linear order in spins we do not need to distinguish between orthogonality to $u_{a}$ or to $p_{a}$ because $p_{a}=m_{a} u_{a}+O\left(s_{a}^{2}\right)$.

4 One could relax this conceptually simplifying condition.

5 To ease the notation, we do not explicitly indicate that the vector $U$ entering the boost operator denotes the local value $U\left(x_{1}\right)$ of the global field $U(x)$.
} 


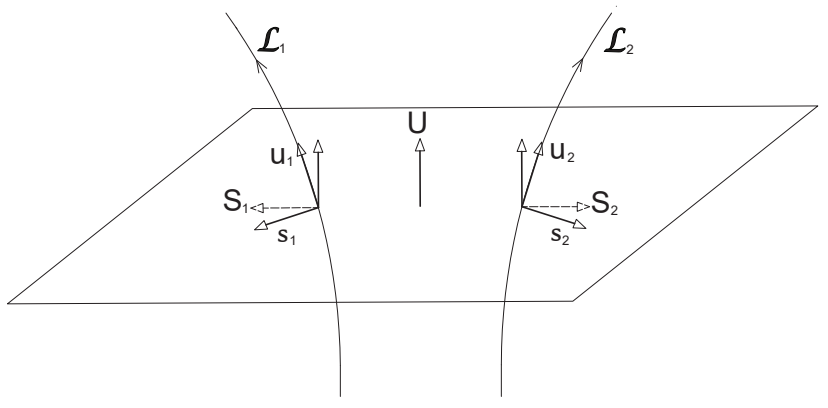

FIG. 1: Schematic representation of the definition of the (abstract) spatial spin vectors $S_{a}(a=1,2)$ orthogonal to the time direction $U$ defining the c.m. frame. They are obtained from the covariant spin vectors $s_{a}$ (orthogonal to $\left.p_{a}=m_{a} u_{a}+O\left(s^{2}\right)\right)$ by the Lorentz boost that rotates $u_{a}$ into the local value of $U$ (drawn vertically), while leaving invariant the 2-plane orthogonal to $u_{a}$ and $U$. For ease of drawing, we have sketched the scattering geometry as if the interaction were repulsive rather than attractive, and we have represented orthogonality relations as if we were in an Euclidean space.

discussed next, only the flat-spacetime asymptotic limit (at $\pm \infty$ ) of $U$ will enter our results.

When decomposed with respect to (wrt) some suitably Cartesianlike orthonormal frame, say $U, E_{1}, E_{2}, E_{3}$, we have $S_{a}=S_{a}^{1} E_{1}+S_{a}^{2} E_{2}+S_{a}^{3} E_{3}$, where the three components $S_{a}^{1}, S_{a}^{2}, S_{a}^{3}$ define the three vector $\mathbf{S}_{a}$. By construction the Euclidean length of $\mathbf{S}_{a}$ is equal to the (constant) $g$-measured length of $s_{a}$, and is therefore also constant

When discussing the scattering operator (12), only the asymptotic values $\left(\right.$ at $\pm \infty$ ) of the spin four-vector $s_{1}$ matter. The corresponding asymptotic values of the spatial spin vector $S_{1}$ are given by

$$
S_{1}^{+}=B_{\eta}\left(u_{1}^{+} \rightarrow U^{\text {as }}\right) s_{1}^{+} ; S_{1}^{-}=B_{\eta}\left(u_{1}^{-} \rightarrow U^{\text {as }}\right) s_{1}^{-} .
$$

They involve a flat Poincaré-Minkowski metric $\eta_{\mu \nu}$, and the common asymptotic value of $U$ at $\pm \infty$ that we denoted as $U^{\text {as }}$.

To end this Section, let us display the (easily computed) explicit value of the general boost linear operator $B_{\eta}(u \rightarrow v)$ in Minkowski space ${ }^{6}$ (for two future-directed timelike unit 4 -vectors $u$ and $v$ ). It reads (suppressing,

6 Actually, if we interpret $u_{\mu}$ as $g_{\mu \nu} u^{\nu}, u \cdot v=g_{\mu \nu} u^{\mu} v^{\nu}$, etc., the formulas below hold in a curved spacetime. for brevity, the subscript $\eta$ )

$$
\begin{gathered}
{[B(u \rightarrow v)]_{\nu}^{\mu}=\delta_{\nu}^{\mu}+\left(u^{\mu}-v^{\mu}\right) u_{\nu}} \\
+\frac{1}{1-u \cdot v}\left(u^{\mu}+v^{\mu}\right)\left[v_{\nu}+(u \cdot v) u_{\nu}\right],
\end{gathered}
$$

or, equivalently,

$$
\begin{aligned}
& {[B(u \rightarrow v)]_{\nu}^{\mu}=\delta_{\nu}^{\mu} } \\
+ & \frac{1}{1-u \cdot v}\left[u^{\mu} u_{\nu}+v^{\mu} v_{\nu}+u^{\mu} v_{\nu}-(1-2 u \cdot v) v^{\mu} u_{\nu}\right] .
\end{aligned}
$$

The Lorentz-boost matrix $B^{\mu}{ }_{\nu} \equiv[B(u \rightarrow v)]_{\nu}^{\mu}$ satisfies $\eta_{\mu \mu^{\prime}} B^{\mu}{ }_{\nu} B^{\mu^{\prime}}{ }_{\nu^{\prime}}=\eta_{\nu \nu^{\prime}}$ and $B^{\mu}{ }_{\nu} u^{\nu}=v^{\mu}$. In addition, when acting on a vector $X^{\mu}$ orthogonal to $u^{\mu}$ (i.e. $u \cdot X=$ $0)$, it transforms it into the vector

$$
B_{\nu}^{\mu} X^{\nu}=X^{\mu}+\frac{(v \cdot X)}{1-(u \cdot v)}\left(u^{\mu}+v^{\mu}\right)
$$

which is orthogonal to $v^{\mu}$. One also easily checks that the inverse matrix of $[B(u \rightarrow v)]^{\mu}{ }_{\nu}$ is $[B(v \rightarrow u)]^{\mu}{ }_{\nu}$. Beware, however, that the naively expected composition rule $B(v \rightarrow w) \circ B(u \rightarrow v)=B(u \rightarrow w)$ is only valid when the third vector $w$ belongs to the 2-plane spanned by $u$ and $v$. [This is linked to the well-known noncommutativity of non-parallel boosts.]

In the following, we shall work, as is standard in EOB theory, in the center-of-mass (c.m.) frame of the binary system. This corresponds to choosing

$$
U^{\text {as }}=\left[\frac{p_{1}+p_{2}}{\mathcal{E}_{\text {real }}}\right]^{+}=\left[\frac{p_{1}+p_{2}}{\mathcal{E}_{\text {real }}}\right]^{-},
$$

where we used the fact that we are considering a conservative dynamics. Here, $\mathcal{E}_{\text {real }}$ denotes the total energy of the binary system (including the rest-mass energy) in the c.m. frame, which is precisely defined as being the (Minkowski) norm of the asymptotic value of $p_{1}+p_{2}$ :

$$
\mathcal{E}_{\text {real }}^{2}=-\left(p_{1}^{+}+p_{2}^{+}\right)^{2}=-\left(p_{1}^{-}+p_{2}^{-}\right)^{2} .
$$

\section{SPIN HOLONOMY}

By combining Eqs. (12), (14) above, we obtain the linear map between the two asymptotic values $($ at $\pm \infty)$ of the spatial spin vector of the first particle, namely

$$
S_{1}^{+}=R_{1} S_{1}^{-},
$$

where

$$
R_{1}=B_{\eta}\left(u_{1}^{+} \rightarrow U^{\mathrm{as}}\right) \Lambda_{1}\left[B_{\eta}\left(u_{1}^{-} \rightarrow U^{\mathrm{as}}\right)\right]^{-1},
$$

or, equivalently,

$$
R_{1}=B_{\eta}\left(u_{1}^{+} \rightarrow U^{\text {as }}\right) \Lambda_{1} B_{\eta}\left(U^{\text {as }} \rightarrow u_{1}^{-}\right),
$$


with a similar result for the second particle. Note that $R_{1}$ is thereby given as the (matrix) product of three $4 \times 4$ matrices.

The linear operator $R_{1}$ is easily seen to leave $U^{\text {as }}$ invariant: $R_{1} U^{\text {as }}=U^{\text {as }}$. In addition, as all the linear maps involved in Eq. (21) preserve the (Minkowski) length, and as $R_{1}$ transforms $S_{1}^{-}$into $S_{1}^{+}$(both spin vectors living in the three-space orthogonal to $U^{\text {as }}$ ), we conclude that the linear map $R_{1}$, that we shall call the spin holonomy of $\mathcal{L}_{1}$, is an $S O(3)$ rotation acting within the three-space orthogonal to $U^{\text {as }}$ (in the asymptotic Minkowski space).

We can express the spin holonomy, Eqs. (21), (22), entirely in terms of the incoming asymptotic values by replacing $u_{1}^{+}$by $\Lambda_{1} u_{1}^{-}$, so that

$$
R_{1}=B_{\eta}\left(\Lambda_{1} u_{1}^{-} \rightarrow U^{\text {as }}\right) \Lambda_{1}\left[B_{\eta}\left(u_{1}^{-} \rightarrow U^{\text {as }}\right)\right]^{-1} .
$$

This is the core theoretical result of our new approach.

\section{POST-MINKOWSKIAN COMPUTATION OF THE SCATTERING AND SPIN HOLONOMIES}

In order to explicitly evaluate the scattering holonomy (11), and the corresponding spin holonomy (23), we need to use a perturbative approach to their computation. We could use PN theory, but as PN theory has already been used to derive in a different way the spin-orbit couplings we are interested in [21, 24, 25], we shall instead use PM theory $9-16]$ to show how it allows one to derive new results, valid to all orders in $v / c$.

At the first PM (1PM) order, i.e. when solving the linearized Einstein equations in harmonic coordinates, the metric generated by our binary system is of the form $g_{\mu \nu}=\eta_{\mu \nu}+h_{\mu \nu}+O\left(G^{2}\right)$, with

$$
h_{\mu \nu}=h_{1 \mu \nu}+h_{2 \mu \nu}
$$

where $h_{1 \mu \nu}$ is generated by $\mathcal{L}_{1}$ and $h_{2 \mu \nu}$ by $\mathcal{L}_{2}$. It is wellknown that, a linear order in $G$, one can neglect self-force effects (see, e.g., [12]). Therefore, when computing the scattering holonomy along $\mathcal{L}_{1}$, the (regularized) metric to be used for computing the parallel transport is simply the contribution $h_{2 \mu \nu}$ from $\mathcal{L}_{2}$. In principle, $h_{2 \mu \nu}$ contains both a contribution proportional to $m_{2}$ and one proportional to $s_{2}$. But, as we are interested in linearin-spin effects, it is enough to include in $h_{2 \mu \nu}$ only the contribution generated by $m_{2}$. Finally, we deal (along $\left.\mathcal{L}_{1}\right)$ with the metric

$$
g_{1 \mu \nu}=\eta_{\mu \nu}+h_{2 \mu \nu} ; \text { with } h_{2 \mu \nu} \propto G m_{2} .
$$

The corresponding value, $\omega_{1}$, of the connection is of order $O(G)$. Working to first order in $G$, we then easily get the value of the scattering holonomy as

$$
\Lambda_{1}=1-\int_{-\infty}^{+\infty} \omega_{1}+O\left(G^{2}\right)
$$

or, more explicitly,

$$
\Lambda_{1 \nu}^{\mu}=\delta_{\nu}^{\mu}-\int_{-\infty}^{+\infty} \omega_{1 \nu}^{\mu}+O\left(G^{2}\right) .
$$

Here,

$$
\omega_{1 \nu}^{\mu}=\eta^{\mu \sigma} \omega_{1 \mu \nu}+O\left(G^{2}\right),
$$

with (going back, for a moment, to an exact expression)

$$
\omega_{1 \mu \nu} \equiv g_{\mu \sigma} \Gamma_{\nu \lambda}^{\sigma} d x^{\lambda}=\frac{1}{2}\left(\partial_{\nu} h_{\mu \lambda}-\partial_{\mu} h_{\nu \lambda}+\partial_{\lambda} h_{\mu \nu}\right) d x^{\lambda}
$$

Note that the last term in this expression for $\omega_{1 \mu \nu}$ is the total derivative $\frac{1}{2} d h_{\mu \nu}(x)$ (evaluated along $\mathcal{L}_{1}$ ), which integrates to zero because of the asymptotic flatness. This leaves an expression for $\omega_{1 \mu \nu}$ which is antisymmetric in $\mu \nu$, and is a curl. We then get

$$
\Lambda_{1 \nu}^{\mu}=\delta_{\nu}^{\mu}+\eta^{\mu \sigma} \theta_{1 \sigma \nu}+O\left(G^{2}\right),
$$

with

$$
\theta_{1 \mu \nu}=+\frac{1}{2} \int_{-\infty}^{+\infty}\left(\partial_{\mu} h_{2 \nu \lambda}-\partial_{\nu} h_{2 \mu \lambda}\right) d x^{\lambda}+O\left(G^{2}\right) \text {. }
$$

Note the antisymmetry of $\theta_{1 \mu \nu}=-\theta_{1 \nu \mu}$, as expected for an infinitesimal $(O(G))$ Lorentz transformation.

Under an $O(G)$ coordinate transformation $x^{\prime \mu}=x^{\mu}-$ $\xi^{\mu}$ (which changes $h_{2 \mu \nu}$ into $h_{2 \mu \nu}+\partial_{\mu} \xi_{\nu}+\partial_{\nu} \xi_{\mu}+O\left(G^{2}\right)$ ), it is easily seen that $\theta_{1 \mu \nu}$ gets the additional term

$$
\begin{aligned}
\Delta \theta_{1 \mu \nu} & =\frac{1}{2} \int_{-\infty}^{+\infty} \partial_{\lambda}\left(\partial_{\mu} \xi_{\nu}-\partial_{\nu} \xi_{\mu}\right) d x^{\lambda}+O\left(G^{2}\right) \\
& =\frac{1}{2}\left[\partial_{\mu} \xi_{\nu}-\partial_{\nu} \xi_{\mu}\right]_{-\infty}^{+\infty} .
\end{aligned}
$$

This vanishes when considering a coordinate transformation $\xi^{\mu}$ which decays at infinity. [More precisely, it suffices that $\partial_{\mu} \xi_{\nu}-\partial_{\nu} \xi_{\mu}$ decays at infinity.] This directly confirms the (geometrically evident) gauge invariance of $\theta_{1 \mu \nu}$.

It is straightforward to compute the 1PM-accurate scattering holonomy $\theta_{1 \mu \nu}$ from the well-known 1PM metric generated by $\mathcal{L}_{2}$. Using, for instance, the results given in Appendices A and B of Ref. [12], and using the simplifying fact that, at this order, we can consider that $\mathcal{L}_{2}$ is a straight worldline (with tangent $u_{2}=u_{2}^{-}$), we have (at an arbitrary field point $x^{\mu}$ )

$$
h_{2 \mu \nu}(x)=2 \frac{G m_{2}}{R_{2}}\left(2 u_{2 \mu} u_{2 \mu}+\eta_{\mu \nu}\right),
$$

where $R_{2}=R_{2}(x)$ denotes the Poincaré-invariant orthogonal distance between the field point $x$ and the straight worldline $\mathcal{L}_{2}\left(=\mathcal{L}_{2}^{-}\right.$, at this order $)$. Explicitly, $R_{2}(x)=\left|x-x_{2}^{\perp}(x)\right|$, where $x_{2}^{\perp}(x)$ denotes the foot of the perpendicular ${ }^{7}$ of the field point $x$ on the line $\mathcal{L}_{2}$.

\footnotetext{
7 One uses here the Poincaré-Minkowski geometry.
} 


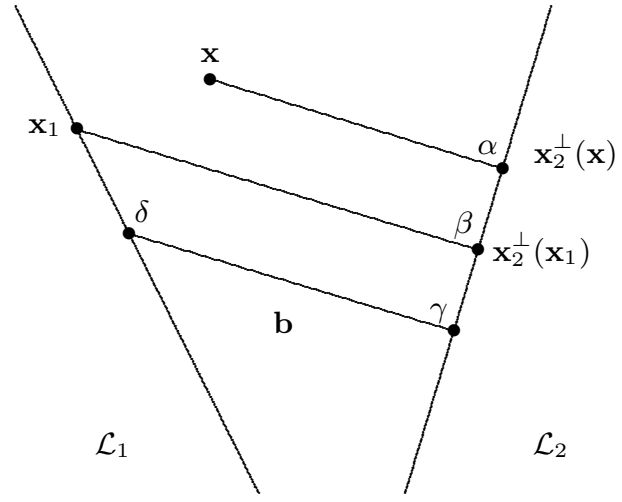

FIG. 2: Geometrical configuration (in Minkowski spacetime) underlying the computation of the scattering holonomy at the $1 \mathrm{PM}$ order. The $1 \mathrm{PM}$ metric generated by $\mathcal{L}_{2}$ is computed at the field point $x$ (which is then made to move all along $\mathcal{L}_{1}$ ). The point $x_{2}^{\perp}(x) \in \mathcal{L}_{2}$ is the foot of the perpendicular drawn from the field point $x$ to $\mathcal{L}_{2}$. The segment $b$ (oriented from $\mathcal{L}_{2}$ towards $\mathcal{L}_{1}$ ) denotes the four-vectorial impact parameter of $\mathcal{L}_{1}$ wrt $\mathcal{L}_{2}$. All the labelled angles $(\alpha, \beta, \gamma, \delta)$ indicate orthogonal incidences (in the Minkowski sense).

[In the case where one must take into account the $O(G)$ curvature of $\mathcal{L}_{2}$ the expression of $h_{2 \mu \nu}(x)$ should involve the half-sum of retarded and advanced tensor potentials generated by $\mathcal{L}_{2}$.] The Poincaré-Minkowski geometrical situation underlying the definition of $x_{2}^{\perp}(x)$ is illustrated in Fig. 2.

The partial derivative wrt $x$ of $h_{2 \mu \nu}(x)$ is then (using again $\dot{u}_{2}=O(G)$ )

$$
\partial_{\lambda} h_{2 \mu \nu}(x)=-2 \frac{G m_{2} R_{2 \lambda}}{R_{2}^{3}}\left(2 u_{2 \mu} u_{2 \nu}+\eta_{\mu \nu}\right)+O\left(G^{2}\right),
$$

where $R_{2}^{\lambda}=x^{\lambda}-x_{2}^{\lambda}(x)$ is the 4 -vector connecting the perpendicular foot $x_{2}^{\perp}(x)$ to $x$.

We must then evaluate along $\mathcal{L}_{1}$ (i.e. replace $x \rightarrow$ $\left.x_{1}\left(\tau_{1}\right)\right)$ the combination of partial derivatives of $h_{2 \mu \nu}(x)$ entering (31), and integrate over $\mathcal{L}_{1}$ using $d x^{\lambda}=u_{1}^{\lambda} d \tau_{1}$. This computation involves the easily evaluated integral (where $x_{1} \equiv x_{1}\left(\tau_{1}\right)$ )

$$
\int_{\mathcal{L}_{1}} d \tau_{1} \frac{x_{1}^{\mu}-x_{2 \perp}^{\mu}\left(x_{1}\right)}{\left|x_{1}-x_{2} \perp\left(x_{1}\right)\right|^{3}}=\frac{2}{\sqrt{\left(u_{1} \cdot u_{2}\right)^{2}-1}} \frac{b^{\mu}}{b^{2}} .
$$

Here, $b^{\mu}$ denotes the Poincaré-invariant four-vectorial impact parameter of $\mathcal{L}_{1}$ wrt $\mathcal{L}_{2}$. We mean by this the value of the four-vector $x_{1}^{\mu}\left(\tau_{1}\right)-x_{2}^{\mu}\left(x_{1}\left(\tau_{1}\right)\right)$ at the moment of closest approach, i.e. for the value of $\tau_{1}$ minimizing $\left|x_{1}-x_{2} \perp\left(x_{1}\right)\right|$. This vectorial impact parameter is simply characterized as being the connecting four-vector $x_{1}^{\mu}-x_{2}^{\mu}$ that is perpendicular both to $u_{1}$ and to $u_{2}$ (see Fig. $2)$. It is convenient to choose the origins of the propertime parameters along $\mathcal{L}_{1}$ and $\mathcal{L}_{2}$ as the end points of $b^{\mu}$ (labelled by $\delta$ and $\gamma$ in Fig. 2), so that we can write the equations of $\mathcal{L}_{1}$ and $\mathcal{L}_{2}$ as: $x_{1}^{\mu}\left(\tau_{1}\right)=x_{1}^{\mu}(0)+u_{1}^{\mu} \tau_{1}$, and $x_{2}^{\mu}\left(\tau_{2}\right)=x_{2}^{\mu}(0)+u_{2}^{\mu} \tau_{2}$, with $x_{1}^{\mu}(0)-x_{2}^{\mu}(0)=b^{\mu}$.
[The vectorial impact parameter of $\mathcal{L}_{2}$ wrt $\mathcal{L}_{1}$ is simply $x_{2}^{\mu}(0)-x_{1}^{\mu}(0)=-b^{\mu}$.]

Inserting Eqs. (34) and (35) into Eq. (31) yields the explicit result

$$
\left(\theta_{1}\right)_{\mu \nu}=-\frac{4 G m_{2} b_{[\mu}}{b^{2} \sqrt{\left(u_{1} \cdot u_{2}\right)^{2}-1}}\left(2\left(u_{1} \cdot u_{2}\right) u_{2 \nu]}+u_{1 \nu]}\right),
$$

where $a_{[\mu} b_{\nu]} \equiv \frac{1}{2}\left(a_{\mu} b_{\nu}-a_{\nu} b_{\mu}\right)$.

Note that this result features the past-directed timelike vector

$$
V_{2}^{\mu} \equiv 2\left(u_{1} \cdot u_{2}\right) u_{2}^{\mu}+u_{1}^{\mu} ;\left(\operatorname{satisfying} V_{2} \cdot V_{2}=-1\right) ;
$$

namely

$$
\left(\theta_{1}\right)_{\mu \nu}=-\frac{2 G m_{2}}{b^{2} \sqrt{\left(u_{1} \cdot u_{2}\right)^{2}-1}}\left(b_{\mu} V_{2 \nu}-b_{\nu} V_{2 \mu}\right) .
$$

Note that while $b^{\mu}$ is antisymmetric under the $1 \leftrightarrow 2$ exchange, $V_{2}$ is asymmetric.

An alternative way of deriving the latter result is to first compute the specialized value of $\left(\theta_{1}\right)_{\mu \nu}$ in the rest-frame of $\mathcal{L}_{2}$ (which means computing the scattering holonomy of a test particle of mass $m_{1}$ moving in the background of a linearized Schwarzschild metric of mass $m_{2}$ ), and to then re-express the result in a manifestly Poincaré-invariant way.

As a first check on the 1PM result, Eqs. (30), (36), (38), let us compute the orbital part of the scattering, i.e. the change in the direction of $\mathcal{L}_{1}$ :

$$
\Delta u_{1} \equiv u_{1}^{+}-u_{1}^{-}=\Lambda_{1} u_{1}^{-}-u_{1}^{-} .
$$

In the following, we will always think of $\Lambda_{1}=\Lambda_{1 \nu}^{\mu}$ as a matrix (an endomorphism of Minkowski spacetime), and use the simplified notation

$$
\Lambda_{1}=1+\theta_{1}+O\left(G^{2}\right),
$$

where 1 denotes the unit matrix (i.e. $\delta_{\nu}^{\mu}$ ), while $\theta_{1}$ will denote the matrix (endomorphism) $\theta_{1 \nu}^{\mu}=\eta^{\mu \sigma} \theta_{1 \sigma \nu}$. With this notation the change

$$
\Delta u_{1}=\theta_{1} u_{1}^{-} \text {; i.e. } \Delta u_{1}^{\mu}=\theta_{1 \nu}^{\mu} u_{1}^{-\nu},
$$

is easily computed from the explicit result (36), (38) to be

$$
\Delta u_{1}^{\mu}=-2 G m_{2} \frac{2\left(u_{1} \cdot u_{2}\right)^{2}-1}{\sqrt{\left(u_{1} \cdot u_{2}\right)^{2}-1}} \frac{b^{\mu}}{b^{2}}
$$

i.e., with $p_{1}^{ \pm}=m_{1} u_{1}^{ \pm}$

$$
\Delta p_{1}^{\mu}=-2 G m_{1} m_{2} \frac{2\left(u_{1} \cdot u_{2}\right)^{2}-1}{\sqrt{\left(u_{1} \cdot u_{2}\right)^{2}-1}} \frac{b^{\mu}}{b^{2}} .
$$

Note that the right-hand side (rhs) of the latter result is antisymmetric under the $1 \leftrightarrow 2$ exchange: $\Delta p_{1}=-\Delta p_{2}$, i.e. conservation of the total four-momentum: $p_{1}^{-}+p_{2}^{-}=$ 
$p_{1}^{+}+p_{2}^{+}$. The result for $\Delta p_{1}$ is easily checked to be in agreement with the known $1 \mathrm{PM}$ scattering angle results; see, e.g., Eqs. (55)-(58) in Ref. [8].

With this definition, and using matrix notation (for endomorphisms) we find that the $1 \mathrm{PM}$ estimate of the spin holonomy, Eq. (23), reads

$$
\begin{aligned}
R_{1} & =B_{\eta}\left(\left(1+\theta_{1}\right) u_{1}^{-} \rightarrow U^{\text {as }}\right)\left(1+\theta_{1}\right)\left[B_{\eta}\left(u_{1}^{-} \rightarrow U^{\text {as }}\right)\right]^{-1} \\
& +O\left(G^{2}\right) .
\end{aligned}
$$

Expanding it to first order in $G$ yields an infinitesimal $S O(3)$ rotation of the form

$$
R_{1}=1+\rho_{1}+O\left(G^{2}\right),
$$

where

$$
\begin{aligned}
\rho_{1} & =B_{\eta}\left(u_{1}^{-} \rightarrow U^{\mathrm{as}}\right) \theta_{1}\left[B_{\eta}\left(u_{1}^{-} \rightarrow U^{\mathrm{as}}\right)\right]^{-1} \\
& +\left(\left(\theta_{1} u_{1}^{-}\right) \frac{\partial}{\partial u_{1}^{-}} B_{\eta}\left(u_{1}^{-} \rightarrow U^{\mathrm{as}}\right)\right)\left[B_{\eta}\left(u_{1}^{-} \rightarrow U^{\mathrm{as}}\right)\right]^{-1} \\
& +O\left(G^{2}\right),
\end{aligned}
$$

belongs to the Lie algebra of spatial rotations in the 3plane orthogonal to $U^{\text {as }}$. Note the second contribution coming from expanding $u_{1}^{+}=\left(1+\theta_{1}\right) u_{1}^{-}$in the argument of the first boost transformation. [In this contribution $\left(\theta_{1} u_{1}^{-}\right) \frac{\partial}{\partial u_{1}^{-}}=\theta_{1}{ }_{\nu}^{\mu} u_{1}^{-\nu} \frac{\partial}{\partial u_{1}^{-\mu}}$ denotes a directional derivative.] The result (46) is entirely expressed in terms of $\theta_{1}$ and of the ingoing four-velocity. Moreover, $\theta_{1}$ can also be expressed (modulo $O\left(G^{2}\right)$ ) entirely in terms of incoming data. [To ease the notation we henceforth suppress the superscripts - on the incoming data.]

As the antisymmetric tensor $\rho_{1 \mu \nu}=\eta_{\mu \sigma} \rho_{1 \nu}^{\sigma}$ is orthogonal to $U_{\text {as }}^{\mu}$, and is easily seen (from the explicit expression of the boost operator (15) and of $\theta_{1}$ ) to be algebraically constructed from the 4 -vectors $b, u_{1}=p_{1} / m_{1}$ and $u_{2}=p_{2} / m_{2}$, it must involve (besides $b$ which, being orthogonal to $u_{1}$ and $u_{2}$, is already orthogonal to $U_{\text {as }}$ ) the projections of $p_{1}$ and $p_{2}$ orthogonally to $U_{\text {as }}$, i.e. the following Minkowski-covariant version of the c.m. momentum:

$$
p_{\text {c.m. }}{ }^{\mu} \equiv\left(\delta^{\mu}{ }_{\nu}+U_{\text {as }}^{\mu} U_{\nu}^{\text {as }}\right) p_{1}^{\nu}=-\left(\delta^{\mu}{ }_{\nu}+U_{\text {as }}^{\mu} U_{\nu}^{\text {as }}\right) p_{2}^{\nu} .
$$

Therefore, $\rho_{1 \mu \nu}$ must be of the form

$$
\rho_{1 \mu \nu}=C_{1} L_{\mu \nu},
$$

where we defined the following covariant version of the orbital angular momentum

$$
L_{\mu \nu}=b_{\mu} p_{\text {c.m. } \nu}-b_{\nu} p_{\text {c.m. } \mu} .
$$

A straightforward calculation of $\rho_{1 \mu \nu}$ obtained by inserting Eq. (38) into Eq. (46), indeed leads to the form (48) with

$$
C_{1}=-\frac{2 G h}{b^{2}(w-1)^{1 / 2}(w+1)^{3 / 2}}\left(\mathrm{c}+\frac{m_{2}}{m_{1}} \mathrm{c}_{*}\right) .
$$

Here, we used the notations,

$$
w \equiv-\left(u_{1} \cdot u_{2}\right) ;(\text { so that } w>0)
$$

for the relative Lorentz $\gamma$ factor between $\mathcal{L}_{1}$ and $\mathcal{L}_{2}$,

$$
h=\frac{\mathcal{E}_{\text {real }}}{M}=\sqrt{1+2 \nu(w-1)},
$$

for a dimensionless measure of the total energy of the system, and

$$
\begin{gathered}
c=\frac{(1+2 w)(h+2 w)-1}{1+h}, \\
\mathbf{c}_{*}=1+2 w .
\end{gathered}
$$

\section{EOB COMPUTATION OF THE SPIN HOLONOMIES}

Let us now see how one can relate the gauge-invariant (three-dimensional) spin holonomies $R_{1}$ and $R_{2}$ to the spin-orbit couplings entering the EOB Hamiltonian. We recall that the (real) EOB Hamiltonian has the form

$$
H\left(\mathbf{R}, \mathbf{P}, \mathbf{S}_{1}, \mathbf{S}_{2}\right)=M \sqrt{1+2 \nu\left(\frac{H_{\mathrm{eff}}}{\mu}-1\right)},
$$

where, at linear order in the spins, the effective EOB Hamiltonian $H_{\text {eff }}$ reads

$$
\begin{aligned}
H_{\mathrm{eff}} & =\sqrt{A\left(\mu^{2}+\mathbf{P}^{2}+\left(\frac{1}{B}-1\right) P_{R}^{2}+Q\right)} \\
& +\frac{G}{R^{3}}\left(g_{S} \mathbf{L} \cdot \mathbf{S}+g_{S_{*}} \mathbf{L} \cdot \mathbf{S}_{*}\right) .
\end{aligned}
$$

Here the functions $A(R)$ and $B(R)$ parametrize the effective metric

$d s_{\text {eff }}^{2}=-A(R) c^{2} d T_{\text {eff }}^{2}+B(R) d R^{2}+R^{2}\left(d \theta^{2}+\sin ^{2} \theta d \phi^{2}\right)$,

$P_{R}$ denotes the EOB radial momentum, while

$$
\mathbf{P}^{2} \equiv P_{R}^{2}+\frac{\mathbf{L}^{2}}{R^{2}}
$$

where $\mathbf{L}$ denotes the EOB orbital angular momentum

$$
\mathbf{L}=\mathbf{R} \times \mathbf{P} .
$$

[Here, and below, we use standard vectorial notation for various EOB vectorlike objects.] In addition, $Q$ in Eq. (56) represents a post-geodesic (Finsler-type) contribution which is at least quartic in momenta. Finally, $\mathbf{S}$ and $\mathbf{S}_{*}$ denote the following symmetric combinations of the two spin vectors

$$
\mathbf{S}=\mathbf{S}_{\mathbf{1}}+\mathbf{S}_{\mathbf{2}} ; \mathbf{S}_{*}=\frac{m_{2}}{m_{1}} \mathbf{S}_{\mathbf{1}}+\frac{m_{1}}{m_{2}} \mathbf{S}_{\mathbf{2}}
$$


while $g_{S}$ and $g_{S_{*}}$ are some corresponding gyrogravitomagnetic ratios (introduced in [21]) which are the focus of the present work.

As shown in Ref. [8], at 1PM order, the post-geodesic term $Q$ is zero, while the effective metric (57) is simply equal to a linearized Schwarzschild metric of mass $M=$ $m_{1}+m_{2}$, i.e.

$$
\begin{aligned}
A(R) & =1-2 \frac{G M}{R}+O\left(G^{2}\right) ; \\
B(R) & =1+2 \frac{G M}{R}+O\left(G^{2}\right) ; \\
Q & =O\left(G^{2}\right) .
\end{aligned}
$$

The two (dimensionless) gyro-gravitomagnetic ratios $g_{S}$ and $g_{S_{*}}$ are functions of $\mathbf{P}^{2} / \mu^{2}, P_{R}^{2} / \mu^{2}, \nu$ and $u \equiv$ $G M / R$. Their current knowledge is the following: (i) their PN expansion is known to the next-to-next-toleading-order level [21, 24, 25]; (ii) the test-mass limit $(\nu \rightarrow 0)$ of the second gyro-gravitomagnetic ratio $g_{S_{*}}$ is known exactly [26, 27]; and (iii) recent self-force computations have allowed one to compute, to high PN order, the circular, and next-to-circular, contributions to $g_{S}$ and $g_{S_{*}}$, see [28 30], and references therein.

Here, we consider the PM expansions of $g_{S}$ and $g_{S_{*}}$, i.e., keeping in mind that $u \equiv G M / R=O(G)$, their expansions in powers of $u$ :

$$
\begin{aligned}
g_{S}\left(\mathbf{P}^{2}, P_{R}^{2}, u\right) & =g_{S}^{1 \mathrm{PM}}\left(\mathbf{P}^{2}, P_{R}^{2}\right)+u g_{S}^{2 \mathrm{PM}}\left(\mathbf{P}^{2}, P_{R}^{2}\right) \\
& +O\left(u^{2}\right) \\
g_{S_{*}}\left(\mathbf{P}^{2}, P_{R}^{2}, u\right) & =g_{S_{*}}^{1 \mathrm{PM}}\left(\mathbf{P}^{2}, P_{R}^{2}\right)+u g_{S_{*}}^{2 \mathrm{PM}}\left(\mathbf{P}^{2}, P_{R}^{2}\right) \\
& +O\left(u^{2}\right) .
\end{aligned}
$$

We have labelled the momentum-dependent, but $u$ independent, leading-order contributions as being of the first PM order because they enter the effective Hamiltonian (56) multiplied by the prefactor $G / R^{3}$.

The equation of motion of the spin vector $\mathbf{S}_{1}$ deduced from the effective Hamiltonian (56) reads

$$
\frac{d \mathbf{S}_{1}}{d T_{\mathrm{eff}}}=\mathbf{\Omega}_{1}^{\mathrm{eff}} \times \mathbf{S}_{1}
$$

where

$$
\mathbf{\Omega}_{1}^{\mathrm{eff}}=\frac{G}{R^{3}} \mathbf{L}\left(g_{S}+\frac{m_{2}}{m_{1}} g_{S_{*}}\right),
$$

and where $T_{\text {eff }}$ is the "effective time" entering (57), i.e. the evolution parameter associated with the dynamics defined by $H_{\text {eff }}$. It differs from the real (coordinate) time $T$ by a factor $d H / d H_{\text {eff }}$ [1]. For our present purpose, we do not need to consider the evolution wrt the real time $T$. Actually, it is convenient to rewrite the evolution equation for $\mathbf{S}_{1}$ in the differential form

$$
d \mathbf{S}_{1}=\boldsymbol{\omega}_{1}^{\mathrm{eff}} \times \mathbf{S}_{1}
$$

where

$$
\begin{aligned}
& \boldsymbol{\omega}_{1}^{\mathrm{eff}}=\boldsymbol{\Omega}_{1}^{\mathrm{eff}} d T_{\mathrm{eff}} \\
= & \frac{G}{R^{3}} \mathbf{L}\left(g_{S}+\frac{m_{2}}{m_{1}} g_{S_{*}}\right) d T_{\mathrm{eff}} .
\end{aligned}
$$

The notation $\boldsymbol{\omega}_{1}^{\text {eff }}$ should not be confused with the notation $\omega_{1}$ used above. Both quantities describe the same physics (the infinitesimal rotation of the spin), but they live in different frameworks.

The evolution equation for $\mathbf{S}_{2}$ is obtained by exchanging everywhere the labels $1 \leftrightarrow 2$.

The spin holonomy of $\mathbf{S}_{1}$ computed in EOB theory is simply obtained by integrating the linear evolution equation (65), i.e.

$$
R_{1}^{\mathrm{EOB}}=T e^{\int_{-\infty}^{+\infty} \boldsymbol{\omega}_{1}^{\mathrm{eff}} \times}
$$

where $\boldsymbol{\omega}_{1}^{\text {eff }} \times$ is viewed as a linear operator (actually an infinitesimal rotation) acting (as $\mathbf{v} \rightarrow \boldsymbol{\omega}_{1}^{\text {eff }} \times \mathbf{v}$ ) in a threedimensional Euclidean vector space .

As $\boldsymbol{\omega}_{1}^{\text {eff }}$ is of order $G$, the $1 \mathrm{PM}$ approximation to the EOB spin holonomy $R_{1}^{E O B}$ is simply

$$
R_{1}^{\mathrm{EOB}}=1+\boldsymbol{\theta}_{1} \times+O\left(G^{2}\right),
$$

where the infinitesimal (i.e. $O(G)$ ) vectorial rotation angle experienced by $\mathbf{S}_{1}$ during the entire scattering is

$$
\begin{aligned}
& \boldsymbol{\theta}_{1}=\int_{-\infty}^{+\infty} \boldsymbol{\omega}_{1}^{\mathrm{eff}} \\
& =\int_{-\infty}^{+\infty} \frac{G}{R^{3}} \mathbf{L}\left(g_{S}^{1 \mathrm{PM}}\left(\mathbf{P}^{2}, P_{R}^{2}\right)+\frac{m_{2}}{m_{1}} g_{S_{*}}^{1 \mathrm{PM}}\left(\mathbf{P}^{2}, P_{R}^{2}\right)\right) d T_{\mathrm{eff}}
\end{aligned}
$$

To turn this result into a fully explicit integral we just need to express $d T_{\text {eff }}$ in terms of dynamical variables. This is obtained by using the equation of motion of $R$ deduced from $H_{\text {eff }}$, namely

$$
\frac{d R}{d T_{\mathrm{eff}}}=\frac{\partial H_{\mathrm{eff}}}{\partial P_{R}}=\frac{A}{B} \frac{P_{R}}{H_{\mathrm{eff}}},
$$

i.e.

$$
d T_{\mathrm{eff}}=\frac{B}{A} H_{\mathrm{eff}} \frac{d R}{P_{R}} .
$$

Actually, as $\boldsymbol{\theta}_{1}$ is of order $G$, we can replace $T_{\text {eff }}$ by its OPM $\left(O\left(G^{0}\right)\right)$ approximation, i.e. use $A \approx 1, B \approx 1$, and

$$
H_{\mathrm{eff}}=\sqrt{\mu^{2}+\mathbf{P}^{2}}+O(G)=\sqrt{\mu^{2}+P_{R}^{2}+\frac{\mathbf{L}^{2}}{R^{2}}}+O(G) .
$$

Moreover, in this approximation the orbital angular momentum $\mathbf{L}$ entering the integral $\boldsymbol{\theta}_{1}$ is constant. We can then use $R$ as integration variable, and compute $P_{R}$ as a function of $R$ and of the 0PM constants of the motion $E_{\text {eff }}=H_{\text {eff }}$ and $\mathbf{L}$ via

$$
P_{R}= \pm \sqrt{E_{\mathrm{eff}}^{2}-\mu^{2}-\frac{\mathbf{L}^{2}}{R^{2}}} .
$$

Finally, we have

$$
\begin{aligned}
\boldsymbol{\theta}_{1} & =G \int_{-\infty}^{+\infty} \frac{\mathbf{L}}{R^{3}}\left(g_{S}^{1 \mathrm{PM}}\left(\mathbf{P}^{2}, P_{R}^{2}\right)+\frac{m_{2}}{m_{1}} g_{S_{*}}^{1 \mathrm{PM}}\left(\mathbf{P}^{2}, P_{R}^{2}\right)\right) \\
& \times \frac{E_{\text {eff }} d R}{P_{R}} .
\end{aligned}
$$


Here, we kept the time-integration limits $-\infty,+\infty$ on the integral sign to indicate the physical integration along the entire motion. The corresponding variation of $R$ goes from $+\infty$ to $R_{\min }$ (with a negative choice for the root defining $P_{R}$ in Eq. (72) ), and then from $R_{\min }$ back to $+\infty$ (with the positive root for $P_{R}$ in Eq. (72)). The net effect is that $\boldsymbol{\theta}_{1}$ can be written as being twice the integral from $R_{\min }$ back to $+\infty$, with a positive value for $P_{R}$.

Let us now remark that the final result for the integrated $O(G)$ spin rotation $\boldsymbol{\theta}_{1}$ involves the two gyro-gravitomagnetic ratios $g_{S}^{1 \mathrm{PM}}\left(\mathbf{P}^{2}, P_{R}^{2}\right)$ and $g_{S_{*}}^{1 \mathrm{PM}}\left(\mathbf{P}^{2}, P_{R}^{2}\right)$ only through the integrals $\int R^{-3} g_{S} d T_{\text {eff }}$ and $\int R^{-3} g_{S_{*}} d T_{\text {eff }}$. Therefore, the physically relevant integrated spin rotation is left invariant under independent $O(G)$ "gauge transformations" of $g_{S}^{1 \mathrm{PM}}\left(\mathbf{P}^{2}, P_{R}^{2}\right)$ and $g_{S_{*}}^{1 \mathrm{PM}}\left(\mathbf{P}^{2}, P_{R}^{2}\right)$ of the type $g_{S}^{\prime}=$ $g_{S} / R^{3}+d f_{S}\left(P_{R}, R, L\right) / d T_{\text {eff }}$, and $g_{S_{*}}^{\prime}=g_{S_{*}} / R^{3}+$ $d f_{S_{*}}\left(P_{R}, R, L\right) / d T_{\text {eff }}$, involving two arbitrary phase-space functions $f_{S}\left(P_{R}, R, L\right), f_{S_{*}}\left(P_{R}, R, L\right)$. [The effectivetime derivatives have to be interpreted as Poisson bracket $\left\{f_{S}, H_{\text {eff }}\right\},\left\{f_{S_{*}}, H_{\text {eff }}\right\}$, evaluated with the effective Hamiltonian.] It is easily checked that this gaugefreedom in the definitions of $g_{S}$ and $g_{S_{*}}$ is the 1PM ana$\log$ of the $O\left(1 / c^{4}\right)$ PN gauge-freedom associated with the canonical transformation Eq. (3.4) in Ref. 21] (and extended to the next PN order in [24, 25]). As remarked in footnote 9 of [21], this gauge-freedom comes from the arbitrariness in the choice of a local frame to measure the spatial spin vectors. In the PN framework, this arbitrariness is parametrized, at each PN order by a finite numer of parameters (see below). In our present PM framework, the arbitrariness is larger because it involves, at each $\mathrm{PM}$ order (say the $n \mathrm{PM}$ level), two functions, which, as is easily seen, can be taken of the form $f_{S}=P_{R} R^{-2} \hat{f}_{S}\left(P_{R}^{2}, L^{2} / R^{2}\right) u^{n-1}$, and $f_{S_{*}}=P_{R} R^{-2} \hat{f}_{S_{*}}\left(P_{R}^{2}, L^{2} / R^{2}\right) u^{n-1}$. In particular, at the $1 \mathrm{PM}$ order $(n=1)$, it is easy to check that this large functional freedom can arbitrarily change the $P_{R}$ dependence of $g_{S}^{1 \mathrm{PM}}\left(\mathbf{P}^{2}, P_{R}^{2}\right)$ and $g_{S_{*}}^{1 \mathrm{PM}}\left(\mathbf{P}^{2}, P_{R}^{2}\right)$.

Ref. [21], having in view the application of EOB theory to quasi-circular inspiralling and coalescing binary black holes, had suggested to simplify the momentum dependence of $g_{S}$ and $g_{S_{*}}$ by making them depend (at each PN order) only on $P_{R}^{2}$ (and not on $\mathbf{P}^{2}$ ). Within our present PM context, it seems technically more convenient to replace the latter "DJS gauge", by an "anti-DJS" gauge where, at the $n \mathrm{PM}$ order, $g_{S}$ and $g_{S_{*}}$ only depend (after factoring the PM prefactor $u^{n-1}$ ) on $\mathbf{P}^{2}$. In particular, at the $1 \mathrm{PM}$ level, this means using $P_{R}$-independent gyro-gravitomagnetic ratios of the form $g_{S}^{1 \mathrm{PM}}\left(\mathbf{P}^{2}\right)$ and $g_{S_{*}}^{1 \mathrm{PM}}\left(\mathbf{P}^{2}\right)$.

Using such an "anti-DJS" gauge, it is very simple to compute the integral (69), because $g_{S}$ and $g_{S_{*}}$ become constant $\left(\mathbf{P}^{2}\right.$ being constant at the $0 \mathrm{PM}$ level) and can be factored out. We have then

$$
\boldsymbol{\theta}_{1}=G E_{\text {eff }} \mathbf{L}\left(g_{S}^{1 \mathrm{PM}}+\frac{m_{2}}{m_{1}} g_{S *}^{1 \mathrm{PM}}\right) \mathcal{I},
$$

where $\mathcal{I}$ denotes the elementary integral

$$
\begin{aligned}
\mathcal{I} & =2 \int_{R_{\min }}^{\infty} \frac{1}{R^{3}} \frac{d R}{\sqrt{E_{\mathrm{eff}}^{2}-\mu^{2}-\frac{\mathbf{L}^{2}}{R^{2}}}} \\
& =\frac{1}{L^{2}} \int_{0}^{x_{\min }} \frac{d x}{\sqrt{x_{\min }-x}}=\frac{2}{L^{2}} \sqrt{x_{\min }}
\end{aligned}
$$

with $x=L^{2} / R^{2}$ and $x_{\min }=E_{\text {eff }}^{2}-\mu^{2}$. This yields the final explicit result

$$
\boldsymbol{\theta}_{1}=2 G E_{\text {eff }} \sqrt{E_{\text {eff }}^{2}-\mu^{2}} \frac{\mathbf{L}}{L^{2}}\left(g_{S}^{1 \mathrm{PM}}\left(\mathbf{P}^{2}\right)+\frac{m_{2}}{m_{1}} g_{S *}^{1 \mathrm{PM}}\left(\mathbf{P}^{2}\right)\right)
$$

\section{DICTIONARY BETWEEN THE PM RESULT AND THE EOB ONE}

One of the defining features of EOB theory is to be able to identify the spatial spin vectors $\mathbf{S}_{1}, \mathbf{S}_{2}$ entering the EOB Hamiltonian with the canonical spatial spin vectors entering the real (PN-expanded, or PM-expanded) dynamics, and also to be able to identify the total c.m. angular momentum of the system $\mathbf{J}$ with the EOB total angular momentum $\mathbf{L}+\mathbf{S}_{1}+\mathbf{S}_{2}$. Because of these identifications, and because the spin vectors become immune to (asymptotic-flatness respecting) gauge ambiguities when considering incoming and outgoing scattering states, the dictionary between the real spin holonomy, and its EOB counterpart, is simply the equality

$$
R_{1}^{\text {real }}=R_{1}^{\mathrm{EOB}} .
$$

When applying this equality in the present case where the lhs is computed within the PM framework as $R_{1}^{\text {real }}=$ $R_{1}^{\mathrm{PM}}=1+\rho_{1}+O\left(G^{2}\right)$, while the rhs is computed as $R_{1}^{\mathrm{EOB}}=1+\boldsymbol{\theta}_{1} \times+O\left(G^{2}\right)$, we simply identify the 3 -space orthogonal to $U$ in which $\rho_{1}$ lives with the 3 -space in which $\boldsymbol{\theta}_{1}$ lives, so that we get

$$
\rho_{1}=\boldsymbol{\theta}_{1} \times+O\left(G^{2}\right) .
$$

In terms of tensor components wrt a 3 -frame this yields

$$
\rho_{1 i j}=-\theta_{1 i j}
$$

From Eq. (48), the lhs involves

$$
\rho_{1 i j}=C_{1} L_{i j}=C_{1}\left(b_{i} p_{\text {c.m. } j}-b_{j} p_{\text {c.m. } i}\right) .
$$

But the definition of the 4 -vectors $b$ and $p_{\text {c.m. have been }}$ chosen so that their spatial projections orthogonal to $U$ entering the above equation are precisely such that $L_{i j}$ are the components of the c.m. orbital angular momentum. Moreover, the definition of the EOB formalism is such that the vector $\mathbf{L}$ entering the EOB Hamiltonian can be identified with the real c.m. angular momentum. [On both sides, PM and EOB, as we talk about linearin-spin effects, we can treat the orbital dynamics as if 
we were treating nonspinning bodies. This allows us to identify ${ }^{8} L_{i j}$ with L.] We therefore see that the condition (79) is compatible with the tensor structure of both sides, and simply leads to an identification of two scalar factors, namely

$$
-C_{1}=\frac{2 G E_{\text {eff }} \sqrt{E_{\mathrm{eff}}^{2}-\mu^{2}}}{L^{2}}\left(g_{S}^{1 \mathrm{PM}}+\frac{m_{2}}{m_{1}} g_{S *}^{1 \mathrm{PM}}\right) .
$$

This gives a condition to determine both $g_{S}^{1 \mathrm{PM}}\left(\mathbf{P}^{2}\right)$ and $g_{S_{*}}^{1 \mathrm{PM}}\left(\mathbf{P}^{2}\right)$. Indeed, we have seen above that $-C_{1}$ had a similar structure $\propto \mathrm{c}+\frac{m_{2}}{m_{1}} \mathrm{c}_{*}$, so that $g_{S}^{1 \mathrm{PM}}\left(\mathbf{P}^{2}\right) \propto \mathrm{c}$ and $g_{S_{*}}^{1 \mathrm{PM}}\left(\mathbf{P}^{2}\right) \propto \mathrm{C}_{*}$.

In order to get the explicit values of $g_{S}^{1 \mathrm{PM}}\left(\mathbf{P}^{2}\right)$ and $g_{S_{*}}^{1 \mathrm{PM}}\left(\mathbf{P}^{2}\right)$ we just need to translate the kinematical PM quantities $w, h$ and $b$ entering $C_{1}$ into their dynamical EOB counterparts. The EOB dictionary (which has been recently proven to be valid to all orders in $v / c$ [8]) yields the simple links (where it is convenient to define $\mathbf{p} \equiv$ $\mathbf{P} / \mu)$ :

$$
w=\frac{E_{\mathrm{eff}}}{\mu}=\frac{1}{\mu} \sqrt{\mu^{2}+\mathbf{P}^{2}}+O(G)=\sqrt{1+\mathbf{p}^{2}}+O(G),
$$

and

$$
h=\frac{\mathcal{E}_{\text {real }}}{M}=\sqrt{1+2 \nu\left(\frac{E_{\mathrm{eff}}}{\mu}-1\right)} .
$$

In addition, Eqs. (51), (52) and (54) in Ref. 8 yield the 8 If we could not neglect spin-orbit corrections to the orbital dynamics we should examine more carefully the expression of the following links between the impact parameter and PM quantities:

$$
L=b p_{\text {c.m. }},
$$

and

$$
\mathcal{E}_{\text {real }} p_{\text {c.m. }}=\mathcal{D}=\sqrt{\left(p_{1} \cdot p_{2}\right)^{2}-p_{1}^{2} p_{2}^{2}}
$$

i.e.

$$
\mathcal{E}_{\text {real }} p_{\text {c.m. }}=m_{1} m_{2} \sqrt{w^{2}-1} .
$$

Note that $|\mathbf{P}|$ is not equal to $p_{\text {c.m. }}$. but rather we have the link

$$
|\mathbf{P}|=h p_{\text {c.m. }} .
$$

Using these links (and remembering the definition $\nu=$ $m_{1} m_{2} / M^{2}$ ) we finally derive the $1 \mathrm{PM}$ values (in the antiDJS gauge) of the EOB gyrogravitomagnetic factors:

$$
\begin{gathered}
g_{S}^{1 \mathrm{PM}}=\frac{\mathrm{c}}{h w(1+w)}=\frac{(1+2 w)(h+2 w)-1}{h w(1+w)(1+h)}, \\
g_{S *}^{1 \mathrm{PM}}=\frac{\mathrm{c}_{*}}{h w(1+w)}=\frac{1+2 w}{h w(1+w)},
\end{gathered}
$$

i.e., explicitly, introducing the shorthand notation,

$$
w_{p} \equiv \sqrt{1+\mathbf{p}^{2}},
$$

total angular momentum $J_{\mu \nu}$ in the real dynamics.

$$
\begin{aligned}
g_{S}^{1 \mathrm{PM}}\left(\mathbf{p}^{2}, \nu\right) & =\frac{\left(1+2 w_{p}\right)\left(\sqrt{1+2 \nu\left(w_{p}-1\right)}+2 w_{p}\right)-1}{w_{p}\left(1+w_{p}\right) \sqrt{1+2 \nu\left(w_{p}-1\right)}\left(1+\sqrt{1+2 \nu\left(w_{p}-1\right)}\right)} \\
g_{S_{*}}^{1 \mathrm{PM}}\left(\mathbf{p}^{2}, \nu\right) & =\frac{\left(1+2 w_{p}\right)}{w_{p}\left(1+w_{p}\right) \sqrt{1+2 \nu\left(w_{p}-1\right)}} .
\end{aligned}
$$

These last two equations are the central new technical results of the present paper.

\section{COMPARISON WITH PREVIOUS RESULTS}

Let us now compare our results for $g_{S}^{1 \mathrm{PM}}\left(\mathbf{p}^{2}, \nu\right)$ and $g_{S_{*}}^{1 \mathrm{PM}}\left(\mathbf{p}^{2}, \nu\right)$ to the previously acquired knowledge of these gyrogravitomagnetic ratios. First, let us recall that, at the linear order in spins at which we are working, and in the anti-DJS gauge we are using, $g_{S}$ and $g_{S_{*}}$ are functions of three (dimensionless) variables, namely $\mathbf{p}^{2}, \nu$ and $u=$ $G M / R$.
In the extreme mass-ratio limit $\nu \rightarrow 0$, one knows the exact values of $g_{S}$ and $g_{S_{*}}$, namely

$$
\lim _{\nu \rightarrow 0} g_{S}\left(\mathbf{p}^{2}, \nu, u\right)=2,
$$

as deduced in Refs. [4, 21] from the Kerr metric, and

$$
\lim _{\nu \rightarrow 0} g_{S_{*}}=\frac{1}{1+W}+\frac{1}{W} \frac{2}{1+\frac{1}{\sqrt{1-2 u}}}
$$

as derived in Refs. [26, 27] (and simplified in Eq. (2.21) 
of [29], and Eq. (4.14) of [30]). Here, $W$ denotes

$$
W=\sqrt{1+\mathbf{p}^{2}-2 u p_{r}^{2}}=\sqrt{1+(1-2 u) p_{r}^{2}+\frac{\mathbf{L}^{2}}{\mu^{2} R^{2}}} .
$$

In the limit $G \rightarrow 0$, i.e. $u \rightarrow 0$ (keeping, however, fixed the centrifugal energy term $\mathbf{L}^{2} /\left(\mu^{2} R^{2}\right)$ which does not contain a factor $G$ ), we get $W=w_{p}$, so that

$$
\lim _{G \rightarrow 0} \lim _{\nu \rightarrow 0} g_{S_{*}}=\frac{1}{1+w_{p}}+\frac{1}{w_{p}}=\frac{\left(1+2 w_{p}\right)}{w_{p}\left(1+w_{p}\right)} .
$$

It is easy to check that the $\nu \rightarrow 0$ limits of our $1 \mathrm{PM}$ results (91), (92), agree with the corresponding expressions (93), (96).

As a second check on our results, let us compare them to the fractionally $O\left(1 / c^{4}\right)$ accurate $\mathrm{PN}$ expansions of $g_{S}$ and $g_{S_{*}}[24,25]$. To make this comparison, we need, how- ever, to use an anti-DJS spin gauge. Let us recall, that the results of Refs. [21, 24, 25] involve some arbitrary gauge parameters. [As already mentioned, this arbitrariness is linked to introducing a time-dependent rotation of the local frame in which the spins are measured 21].] From Eqs. (29), (30) in [24], we have the structure

$$
\begin{aligned}
g_{S}^{\mathrm{PN}} & =2+\frac{1}{c^{2}} g_{S}^{\mathrm{NLO}}(a)+\frac{1}{c^{4}} g_{S}^{\mathrm{NNLO}}(a ; \alpha, \beta, \gamma), \\
g_{S_{*}}^{\mathrm{PN}} & =\frac{3}{2}+\frac{1}{c^{2}} g_{S}^{\mathrm{NLO}}(b)+\frac{1}{c^{4}} g_{S^{*}}^{\mathrm{NNLO}}(b ; \delta, \zeta, \eta),
\end{aligned}
$$

where the gauge parameters entering $g_{S}$ are called $a$ (which enters at $O\left(1 / c^{2}\right)$ ) and $\alpha, \beta, \gamma$ (entering at $\left.O\left(1 / c^{4}\right)\right)$, while the corresponding gauge parameters entering $g_{S_{*}}$ are called $b$ (which enters at $O\left(1 / c^{2}\right)$ ) and $\delta, \zeta, \eta$ (entering at $O\left(1 / c^{4}\right)$ ). The explicit form of these $\mathrm{PN}$ expansions read

$$
\begin{aligned}
g_{S}^{\mathrm{PN}}=2 & \left.+\frac{1}{c^{2}}\left[\left(\frac{3}{8} \nu+a\right) \mathbf{p}^{2}-\left(\frac{9}{2} \nu+3 a\right)(\mathbf{n} \cdot \mathbf{p})^{2}\right)-u(\nu+a)\right] \\
& +\frac{1}{c^{4}}\left[-u^{2}\left(9 \nu+\frac{3}{2} \nu^{2}+a+\alpha\right)\right. \\
& +u\left[(\mathbf{n} \cdot \mathbf{p})^{2}\left(\frac{35}{4} \nu-\frac{3}{16} \nu^{2}+6 a-4 \alpha-3 \beta-2 \gamma\right)+\mathbf{p}^{2}\left(-\frac{17}{4} \nu+\frac{11}{8} \nu^{2}-\frac{3 a}{2}+\alpha-\gamma\right)\right] \\
& +\left(\frac{9}{4} \nu-\frac{39}{16} \nu^{2}+\frac{3 a}{2}+3 \beta-3 \gamma\right) \mathbf{p}^{2}(\mathbf{n} \cdot \mathbf{p})^{2}+\left(\frac{135}{16} \nu^{2}-5 \beta\right)(\mathbf{n} \cdot \mathbf{p})^{4} \\
& \left.+\left(-\frac{5}{8} \nu-\frac{a}{2}+\gamma\right) \mathbf{p}^{4}\right], \\
g_{S_{*}}^{\mathrm{PN}}=\frac{3}{2} & +\frac{1}{c^{2}}\left[\left(-\frac{5}{8}+\frac{1}{2} \nu+b\right) \mathbf{p}^{2}-\left(\frac{15}{4} \nu+3 b\right)(\mathbf{n} \cdot \mathbf{p})^{2}-u\left(\frac{1}{2}+\frac{5}{4} \nu+b\right)\right] \\
& +\frac{1}{c^{4}}\left[-u^{2}\left(\frac{1}{2}+\frac{55}{8} \nu+\frac{13}{8} \nu^{2}+b+\delta\right)\right. \\
& +u\left[(\mathbf{n} \cdot \mathbf{p})^{2}\left(\frac{5}{4}+\frac{109}{8} \nu+\frac{3}{4} \nu^{2}+6 b-4 \delta-3 \zeta-2 \eta\right)+\mathbf{p}^{2}\left(\frac{1}{4}-\frac{59}{16} \nu+\frac{3}{2} \nu^{2}-\frac{3 b}{2}+\delta-\eta\right)\right] \\
& +\left(\frac{57}{16} \nu-\frac{21}{8} \nu^{2}+\frac{3 b}{2}+3 \zeta-3 \eta\right) \mathbf{p}^{2}(\mathbf{n} \cdot \mathbf{p})^{2}+\left(\frac{15}{2} \nu^{2}-5 \zeta\right)(\mathbf{n} \cdot \mathbf{p})^{4} \\
& \left.+\left(\frac{7}{16}-\frac{11}{16} \nu-\frac{\nu^{2}}{16}-\frac{b}{2}+\eta\right) \mathbf{p}^{4}\right] .
\end{aligned}
$$

It is easily checked that we can move to an anti-DJS gauge (i.e. eliminate the dependence of $g_{S}$ and $g_{S *}$ on $p_{r}$ to keep a dependence only on $\mathbf{p}^{2}$ and $u$ ), by the following choice of gauge parameters:

$$
\begin{array}{ll}
a & =-\frac{3}{2} \nu, \quad \alpha=-\frac{1}{16} \nu-\frac{7}{4} \nu^{2}, \quad \beta=\frac{27}{16} \nu^{2}, \quad \gamma=\frac{7}{8} \nu^{2}, \\
b & =-\frac{5}{4} \nu, \quad \eta=\frac{9}{16} \nu+\frac{5}{8} \nu^{2}, \quad \delta=\frac{5}{16}+\frac{5}{4} \nu-\frac{5}{4} \nu^{2}, \quad \zeta=\frac{3}{2} \nu^{2} .
\end{array}
$$


This yields the following results,

$$
\begin{aligned}
g_{S}^{\mathrm{PN}} & =2+\frac{1}{c^{2}}\left(-\frac{9}{8} \nu \mathbf{p}^{2}+\frac{1}{2} u \nu\right) \\
& +\frac{1}{c^{4}}\left[-u^{2}\left(\frac{119}{16} \nu-\frac{1}{4} \nu^{2}\right)+u \mathbf{p}^{2}\left(-\frac{33}{16} \nu-\frac{5}{4} \nu^{2}\right)+\left(\frac{1}{8} \nu+\frac{7}{8} \nu^{2}\right) \mathbf{p}^{4}\right] \\
g_{S_{*}}^{\mathrm{PN}} & =\frac{3}{2}+\frac{1}{c^{2}}\left[\left(-\frac{5}{8}-\frac{3}{4} \nu\right) \mathbf{p}^{2}-\frac{1}{2} u\right] \\
& +\frac{1}{c^{4}}\left[-u^{2}\left(\frac{13}{16}+\frac{55}{8} \nu+\frac{3}{8} \nu^{2}\right)+u \mathbf{p}^{2}\left(\frac{9}{16}-\frac{9}{8} \nu-\frac{3}{8} \nu^{2}\right)+\left(\frac{7}{16}+\frac{1}{2} \nu+\frac{9}{16} \nu^{2}\right) \mathbf{p}^{4}\right] .
\end{aligned}
$$

The PM re-expansion of these (PN-expanded) expressions amounts to ordering them in powers of $u=G M / R$, i.e.

$$
\begin{aligned}
g_{S} & =g_{S}^{1 \mathrm{PM}}\left(\mathbf{p}^{2}\right)+u g_{S}^{2 \mathrm{PM}}\left(\mathbf{p}^{2}\right)+u^{2} g_{S}^{3 \mathrm{PM}}\left(\mathbf{p}^{2}\right), \\
g_{S_{*}} & =g_{S_{*}}^{1 \mathrm{PM}}\left(\mathbf{p}^{2}\right)+u g_{S_{*}}^{2 \mathrm{PM}}\left(\mathbf{p}^{2}\right)+u^{2} g_{S_{*}}^{3 \mathrm{PM}}\left(\mathbf{p}^{2}\right),(10
\end{aligned}
$$

with

$$
\begin{aligned}
g_{S}^{1 \mathrm{PM}}\left(\mathbf{p}^{2}\right)= & 2-\frac{9}{8 c^{2}} \nu \mathbf{p}^{2}+\frac{1}{c^{4}}\left(\frac{1}{8} \nu+\frac{7}{8} \nu^{2}\right) \mathbf{p}^{4} \\
& +O\left(\mathbf{p}^{6}\right) \\
g_{S_{*}}^{1 \mathrm{PM}}\left(\mathbf{p}^{2}\right)= & \frac{3}{2}+\frac{1}{c^{2}}\left(-\frac{5}{8}-\frac{3}{4} \nu\right) \mathbf{p}^{2} \\
& +\frac{1}{c^{4}}\left(\frac{7}{16}+\frac{1}{2} \nu+\frac{9}{16} \nu^{2}\right) \mathbf{p}^{4} \\
& +O\left(\mathbf{p}^{6}\right), \\
g_{S}^{2 \mathrm{PM}}\left(\mathbf{p}^{2}\right)= & \frac{1}{2 c^{2}} \nu+\frac{1}{c^{4}} \mathbf{p}^{2}\left(-\frac{33}{16} \nu-\frac{5}{4} \nu^{2}\right)+O\left(\mathbf{p}^{4}\right), \\
g_{S_{*}}^{2 \mathrm{PM}}\left(\mathbf{p}^{2}\right)= & -\frac{1}{2 c^{2}}+\frac{1}{c^{4}} \mathbf{p}^{2}\left(\frac{9}{16}-\frac{9}{8} \nu-\frac{3}{8} \nu^{2}\right) \\
+ & O\left(\mathbf{p}^{4}\right),
\end{aligned}
$$

and

$$
\begin{aligned}
g_{S}^{3 \mathrm{PM}}\left(\mathbf{p}^{2}\right) & =\frac{1}{c^{4}}\left(-\frac{119}{16} \nu+\frac{1}{4} \nu^{2}\right)+O\left(\mathbf{p}^{2}\right), \\
g_{S *}^{3 \mathrm{PM}}\left(\mathbf{p}^{2}\right) & =\frac{1}{c^{4}}\left(-\frac{13}{16}-\frac{55}{8} \nu-\frac{3}{8} \nu^{2}\right) \\
& +O\left(\mathbf{p}^{2}\right) .
\end{aligned}
$$

It is straightforward to check that the PN expansion (i.e. the expansion in powers of $\mathbf{p}^{2}$ ) of our exact $1 \mathrm{PM}$ results (91), (92), agree with the PN results (104) (within their $O\left(\mathbf{p}^{6}\right)$ accuracy).

Let us finally briefly mention the knowledge of the gravitational self-force (SF) expansion (i.e. the expansion in powers of $\nu$ ) of $g_{S}$ and $g_{S_{*}}$. Several recent papers (notably Refs. 28 30]) have investigated the first term $(\propto \nu)$ in the SF expansion of $g_{S}$ and $g_{S_{*}}$. However, these
SF studies have been limited to the case of circular, or slightly-eccentric bound orbits. Because of this fact, one cannot directly compare our close-to-hyperbolic results to such SF results. Let us, however, mention an interesting aspect of our 1PM results (91), (92), namely their behavior at ultrarelativistic energies. First, let us remark that, in the extreme-mass-ratio limit $\nu \rightarrow 0$, we have the following large-energy behavior when the relative Lorentz $\gamma$ factor $w=\sqrt{1+\mathbf{p}^{2}}$ tends to $\infty$ :

$$
\left[g_{S}\right]_{\nu=0}=2 ;\left[g_{S_{*}}\right]_{\nu=0} \sim \frac{2}{w} ; \text { as } w \rightarrow \infty .
$$

By contrast, in the comparable-mass case, i.e. when $\nu \neq$ 0 , we have the large-energy behaviors

$$
g_{S} \sim \frac{2}{\nu w} ; g_{S_{*}} \sim \frac{\sqrt{2}}{\sqrt{\nu} w^{3 / 2}} ; \text { as } w \rightarrow \infty .
$$

In words, this means that comparable-mass effects drastically change the large-energy behavior of $g_{S}$ and $g_{S_{*}}$. When $\nu \neq 0$, both gyrogravitomagnetic ratios tend to zero at large energies, and $g_{S_{*}}$ tend to zero faster than before. The large-energy behavior of $g_{S}$ and $g_{S_{*}}$ (both for $\nu=0$ and for $\nu \neq 0$ ) are illustrated in Fig. 3 .

This illustrates the singular character of the SF expansion at large energies. Actually, we see on Eqs. (91), (92) that the extra $\nu$-related factors embodying these faster large-energy decays consists of a factor $\sim$ $1 /(1+2 \nu(w-1))$ in $g_{S}$, and a factor $\sim 1 / \sqrt{1+2 \nu(w-1)}$ in $g_{S_{*}}$. Performing the formal SF expansion of $g_{S}$ and $g_{S_{*}}$ would mean expanding these factors in powers of $\nu$, according to, e.g.

$$
\frac{1}{\sqrt{1+2 \nu(w-1)}}=1-\nu(w-1)+O\left(\nu^{2}\right) .
$$

We see that, while the exact PM-type lhs goes to zero as $w \rightarrow+\infty$, its first-order SF-expanded version on the rhs goes towards $-\infty$ as $w \rightarrow+\infty$. Such a singular behavior of SF-expanded quantities was first observed in Ref. 31] for orbital effects, and was also found for spin-orbit effects in Refs. [28, 29]. However, in the SF context, the largeenergy regime is reached when considering orbits near the light-ring of a black hole. As the latter near-light-ring 


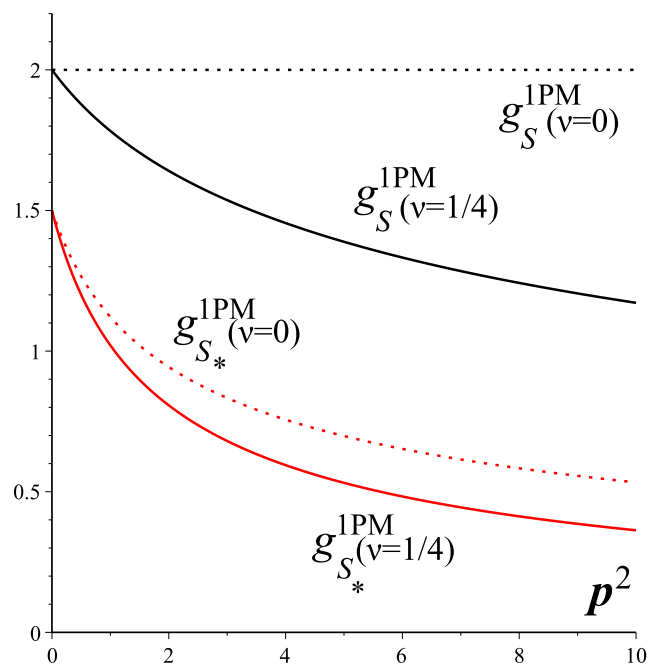

FIG. 3: Our 1PM results, Eqs. (91), (92), for the EOB spinorbit couplings $g_{S}^{1 \mathrm{PM}}\left(\mathbf{p}^{2}, \nu\right)$ and $g_{S *}^{1 \mathrm{PM}}\left(\mathbf{p}^{2}, \nu\right)$ are plotted as functions of $\mathbf{p}^{2}$ for the two limiting values of the symmetric mass-ratio $\nu=0$ and $\nu=\frac{1}{4}$.

limit mixes large-kinetic-energy effects with strong-field effects, one cannot directly translate our specific largeenergy PM effects into a corresponding light-ring behavior. Still, we think that our PM approach illuminates the issue by showing the explicit appearance of factors involving powers of $1 / \sqrt{1+2 \nu(w-1)}$, which generate singular SF terms of the type shown on the rhs of Eq. (109).

\section{CONCLUSIONS}

A new gauge-invariant approach to the description of spin-orbit coupling in binary systems has been introduced. It is based on the new, related, concepts of "scattering holonomy" (integrated connection along an entire hyperbolic-motion worldline), and "spin holonomy" (action of the scattering holonomy on the spatial spin threevector). We have formulated our approach in the approximation where we neglected spin-curvature effects (corresponding, in the Hamiltonian approach, to nonlinearin-spin effects), but it can be generalized (by modifying the evolution law of the spins) to the inclusion of spin-curvature effects. Compared to the approach (suggested in [8]) consisting in including spin-orbit effects in the computation of the scattering angle, the approach presented here has the significant advantage that we can derive information on spin-orbit effects from a calculation where we actually neglect spin-effects ! [In that respect, this is akin to the method used in Refs. 20, 21].]

We have applied here our method to the explicit computation of the spin-orbit couplings at the first order in the post-Minkowskian expansion (first order in $G$ and all orders in $v / c$ ). Using then an extension of the EOB/real dynamics dictionary, we have transcribed our results into the computation of the two gyrogravitomagnetic ratios $g_{S}$ and $g_{S_{*}}$, see Eqs. (91), (92). Our results are compatible with the previous knowledge on these coupling coefficients, but extend our knowledge in the direction of arbitrarily-high momenta. In particular, it has been found that, for comparable-mass binary systems $(\nu \neq 0)$, $g_{S}^{1 \mathrm{PM}}\left(\mathbf{p}^{2}, \nu\right)$ and $g_{S *}^{1 \mathrm{PM}}\left(\mathbf{p}^{2}, \nu\right)$ tend to zero in the ultrarelativistic limit (see Fig. 3). Our work provides new insights on the singular nature of the self-force expansion. We leave to future work the exploitation of our results in the currently physically more urgent case of black-hole coalescences (ellipticlike motions, instead of the hyperboliclike ones considered here). Our all-ordersin- $(v / c)$ results can suggest new ways of resumming the spin-orbit couplings. Let us note that our finding that $g_{S}$ and $g_{S_{*}}$ decay at large kinetic energies, resonate with the finding that the fitting of EOB theory to numerical relativity data indicates a significant decay of $g_{S}$ and $g_{S_{*}}$ during the strong-field coalescence of binary black holes (see, e.g., the calibration of the spin-orbit parameters $d_{\mathrm{SO}}$ in [32, 33], and $c_{3}$ in Refs. [34, 35]). It will be interesting to extend our results to the second postMinkowskian level $\left(O\left(G^{2}\right)\right)$ to complete our information about the regime where both kinetic and the binding energies become large.

\section{Acknowledgments}

We are grateful to Ulysse Schildge for fruitful exchanges during many years. D.B. thanks the International Center for Relativistic Astrophysics Network (ICRANet) and the Italian Istituto Nazionale di Fisica Nucleare (INFN) for partial support and the Institut des Hautes Etudes Scientifiques (IHES) for warm hospitality at various stages during the development of the present project.
[1] A. Buonanno and T. Damour, "Effective one-body approach to general relativistic two-body dynamics," Phys. Rev. D 59, 084006 (1999) gr-qc/9811091.

[2] A. Buonanno and T. Damour, "Transition from inspiral to plunge in binary black hole coalescences," Phys. Rev. D 62, 064015 (2000) gr-qc/0001013.
[3] T. Damour, P. Jaranowski and G. Schäfer, "On the determination of the last stable orbit for circular general relativistic binaries at the third post-Newtonian approximation," Phys. Rev. D 62, 084011 (2000) gr-qc/0005034.

[4] T. Damour, "Coalescence of two spinning black holes: an effective one-body approach," Phys. Rev. D 64, 124013 
(2001) gr-qc/0103018.

[5] B. P. Abbott et al. [LIGO Scientific and Virgo Collaborations], "Observation of Gravitational Waves from a Binary Black Hole Merger," Phys. Rev. Lett. 116, 061102 (2016) arXiv:1602.03837 [gr-qc]].

[6] B. P. Abbott et al. [LIGO Scientific and Virgo Collaborations], "GW151226: Observation of Gravitational Waves from a 22-Solar-Mass Binary Black Hole Coalescence," Phys. Rev. Lett. 116, no. 24, 241103 (2016) arXiv:1606.04855 [gr-qc]].

[7] B. P. Abbott et al. [LIGO Scientific and VIRGO Collaborations], "GW170104: Observation of a 50Solar-Mass Binary Black Hole Coalescence at Redshift 0.2," Phys. Rev. Lett. 118 (2017) no.22, 221101. doi:10.1103/PhysRevLett.118.221101

[8] T. Damour, "Gravitational scattering, post-Minkowskian approximation and Effective One-Body theory," Phys. Rev. D 94, no. 10, 104015 (2016) arXiv:1609.00354 [grqc]].

[9] K. Westpfahl and M. Goller, "Gravitational Scattering Of Two Relativistic Particles In Postlinear Approximation," Lett. Nuovo Cim. 26, 573 (1979).

[10] M. Portilla, "Momentum And Angular Momentum Of Two Gravitating Particles," J. Phys. A 12, 1075 (1979).

[11] M. Portilla, "Scattering Of Two Gravitating Particles: Classical Approach," J. Phys. A 13, 3677 (1980).

[12] L. Bel, T. Damour, N. Deruelle, J. Ibanez and J. Martin, "Poincar-invariant gravitational field and equations of motion of two pointlike objects: The postlinear approximation of general relativity," Gen. Rel. Grav. 13, 963 (1981).

[13] T. Damour and N. Deruelle, "Radiation Reaction and Angular Momentum Loss in Small Angle Gravitational Scattering," Phys. Lett. A 87, 81 (1981).

[14] K. Westpfahl, "High-Speed Scattering of Charged and Uncharged Particles in General Relativity," Fortschr. Physik 33, 417 (1985).

[15] K. Westpfahl, R. Möhles and H Simonis "Energymomentum conservation for gravitational two-body scattering in the post-linear approximation," Classical and Quantum Gravity, 4, L185 (1987).

[16] T. Ledvinka, G. Schäfer and J. Bicak, "Relativistic Closed-Form Hamiltonian for Many-Body Gravitating Systems in the Post-Minkowskian Approximation," Phys. Rev. Lett. 100, 251101 (2008) arXiv:0807.0214 [gr-qc]].

[17] T. Damour, "Gravitational Self Force in a Schwarzschild Background and the Effective One Body Formalism," Phys. Rev. D 81, 024017 (2010) arXiv:0910.5533 [grqc]].

[18] T. Damour, F. Guercilena, I. Hinder, S. Hopper, A. Nagar and L. Rezzolla, "Strong-Field Scattering of Two Black Holes: Numerics Versus Analytics," Phys. Rev. D 89, no. 8, 081503 (2014) arXiv:1402.7307 [gr-qc]].

[19] D. Bini and T. Damour, "Gravitational scattering of two black holes at the fourth post-Newtonian approximation," arXiv:1706.06877 [gr-qc].

[20] T. Damour, P. Jaranowski and G. Schaefer, "Hamiltonian of two spinning compact bodies with next-to-leading order gravitational spin-orbit coupling," Phys. Rev. D 77, 064032 (2008) arXiv:0711.1048 [gr-qc]].

[21] T. Damour, P. Jaranowski and G. Schaefer, "Effective one body approach to the dynamics of two spinning black holes with next-to-leading order spin-orbit cou- pling," Phys. Rev. D 78, 024009 (2008) arXiv:0803.0915 [gr-qc]].

[22] W. G. Dixon, "Dynamics of extended bodies in general relativity. I. Momentum and angular momentum," Proc. Roy. Soc. Lond. A 314, 499 (1970).

[23] F. J. Dyson, "The Radiation theories of Tomonaga, Schwinger, and Feynman," Phys. Rev. 75, 486 (1949).

[24] A. Nagar, "Effective one body Hamiltonian of two spinning black-holes with next-to-next-to-leading order spin-orbit coupling," Phys. Rev. D 84, 084028 (2011) Erratum: [Phys. Rev. D 88, no. 8, 089901 (2013)] doi:10.1103/PhysRevD.84.084028, 10.1103/PhysRevD.88.089901 arXiv:1106.4349 [gr-qc]].

[25] E. Barausse and A. Buonanno, "Extending the effectiveone-body Hamiltonian of black-hole binaries to include next-to-next-to-leading spin-orbit couplings," Phys. Rev. D 84, 104027 (2011) doi:10.1103/PhysRevD.84.104027 arXiv:1107.2904 [gr-qc]].

[26] E. Barausse, E. Racine and A. Buonanno, "Hamiltonian of a spinning test-particle in curved spacetime," Phys. Rev. D 80, 104025 (2009) Erratum: [Phys. Rev. D 85, 069904 (2012)] arXiv:0907.4745 [gr-qc]].

[27] E. Barausse and A. Buonanno, "An Improved effective-one-body Hamiltonian for spinning blackhole binaries," Phys. Rev. D 81, 084024 (2010) doi:10.1103/PhysRevD.81.084024 arXiv:0912.3517 [grqc]].

[28] D. Bini and T. Damour, "Two-body gravitational spinorbit interaction at linear order in the mass ratio," Phys. Rev. D 90, no. 2, 024039 (2014) arXiv:1404.2747][gr-qc]].

[29] D. Bini, T. Damour and A. Geralico, "Spin-dependent two-body interactions from gravitational self-force computations," Phys. Rev. D 92, no. 12, 124058 (2015) Erratum: [Phys. Rev. D 93, no. 10, 109902 (2016)] doi:10.1103/PhysRevD.93.109902, 10.1103/PhysRevD.92.124058 [arXiv:1510.06230 [gr-qc]].

[30] C. Kavanagh, D. Bini, T. Damour, S. Hopper, A. C. Ottewill and B. Wardell, "Spin-orbit precession along eccentric orbits for extreme mass ratio black hole binaries and its effective-one-body transcription," arXiv:1706.00459 [gr-qc].

[31] S. Akcay, L. Barack, T. Damour and N. Sago, "Gravitational self-force and the effective-one-body formalism between the innermost stable circular orbit and the light ring," Phys. Rev. D 86, 104041 (2012) doi:10.1103/PhysRevD.86.104041 arXiv:1209.0964 [grqc]].

[32] A. Taracchini et al., "Prototype effective-one-body model for nonprecessing spinning inspiral-mergerringdown waveforms," Phys. Rev. D 86, 024011 (2012) arXiv:1202.0790 [gr-qc]].

[33] A. Taracchini et al., "Effective-one-body model for blackhole binaries with generic mass ratios and spins," Phys. Rev. D 89, no. 6, 061502 (2014) arXiv:1311.2544 [gr-qc]].

[34] T. Damour and A. Nagar, "New effective-one-body description of coalescing nonprecessing spinning blackhole binaries," Phys. Rev. D 90, no. 4, 044018 (2014) arXiv:1406.6913 [gr-qc]].

[35] A. Nagar, T. Damour, C. Reisswig and D. Pollney, "Energetics and phasing of nonprecessing spinning coalescing black hole binaries," Phys. Rev. D 93, no. 4, 044046 (2016) arXiv:1506.08457 [gr-qc]]. 